\title{
Metodología de trabajo inclusiva en una zona de exclusión social. Compensación educativa e Interculturalidad
}

\author{
Ana Amaro Agudo \\ Universidad de Granada \\ anaamaro@eulainmaculada.com
}

\section{Resumen:}

La ley, para garantizar el derecho fundamental a la educación de todos los alumnos y alumnas, ordena la atención educativa del alumnado con Necesidades Específicas de Apoyo Educativo asociadas a condiciones sociales desfavorecidas, con un conjunto de medidas y actuaciones de carácter compensador. La sociedad actual demanda un cambio en las aulas y ejemplo de ello se recoge en este artículo con la metodología inclusiva llevada a cabo en el Colegio Luisa de Marillac de la ciudad de Granada (España).

Palabras Clave: Compensación educativa; interculturalidad; inclusión; Proyecto Roma

\section{Work inclusive methodology in a social exclusion zone. Educational compensation and Interculturality.}

\begin{abstract}
:
The law, to guarantee the fundamental right to education of all students, the educational attention for students with Specific Needs of Educational Support associated to disadvantaged social conditions with a set of steps and actions of acompensating nature. Today's society demands a change in classrooms and example of it has been collected in this article with the inclusive methodology carried out in Luisa de Marillac Centerin the city of Granada (Spain).
\end{abstract}

Key Words: Educational compensation; Interculturality; Inclusion; Rome Project.

\section{Referencia normalizada:}

Amaro Agudo, A. (2014): Metodología de trabajo inclusiva en una zona de exclusión social. Compesación educativa e interculturalidad. Historia y Comunicación Social. Vol. 19. Núm. Especial Marzo. Págs. 649-659.

Sumario: 1. Introducción. 2. Metodología. 3. Legislación. 4. Educación intercultural y escuela inclusiva. 5. Ejemplo de cultura inclusiva. Colegio Luisa de Marillac (Granada). 5.1. Organización del trabajo dentro del aula. 5.2. Estrategias de enseñanza y metodología de trabajo. 6. Resultados. 


\section{Introducción}

Los centros docentes que escolaricen alumnado con necesidades de compensación educativa podrán elaborar un Plan de Compensación Educativa con el fin de adaptar el currículo a las necesidades y características del mismo, que deberá ser aprobado por la Consejería de Educación. Se reafirma la idea de practicar una educación que llegue a todos y de la que todo el alumnado sin distinción sea partícipe. Sin discriminación por su cultura de origen.

A lo largo de los años esta idea de trabajar la educación por y para todos se ha hecho más fuerte. Asentando sus premisas en pilares como la educación inclusiva. Dando lugar al nacimiento de políticas interculturales y de inclusión, basadas en metodologías de trabajo del profesorado para poder hacer realidad las mismas.

Existen profesionales entusiasmados con su profesión y dispuestos a dar y formarse continuamente para llevar a sus aulas metodologías y estrategias que ayuden a la igualdad en educación.

Ejemplo de ello es el Colegio Luisa de Marillac, situado en una de las zonas más desfavorecidas de la ciudad de Granada (España), con la totalidad de alumnado de etnia gitana y rumana. Asentado en la zona norte de la ciudad de Granada, es un centro de Educación Compensatoria que nace para paliar y solucionar la situación de desarraigo de varias familias desahuciadas y con graves problemas económicos. Las líneas de actuación en el centro han estado marcadas por el trabajo por y para el alumnado del mismo, el profesorado siempre ha estado preocupado por conseguir la máxima inclusión de los mismos en el colegio y en la sociedad, en su entorno. De ahí su adhesión a la metodología del Proyecto Roma y en la actualidad a Comunidades de Aprendizaje.

\section{Metodología}

El diseño y la estructura del artículo lo clasifican dentro de la metodología cualitativa y concretamente al estudio de casos. El estudio de casos "se caracteriza porque presta especial atención a cuestiones que específicamente pueden ser conocidas a través de casos" (Buendía y Colás, 1997:257).

El fundamento del estudio realizado es ejemplificar, a través del caso concreto del colegio Luisa de Marillac, la posibilidad del cambio metodológico llevando a cabo metodologías de trabajo inclusivas como es el caso del Proyecto Roma.

El estudio de casos instrumentales "pretende aportar luz sobre algunas cuestiones o el refinamiento de una teoría...la elección del caso se realiza para avanzar en la comprensión de aquello que nos interesa" Buendía y Colás(1997:257), como sería nuestro estudio en concreto. Con la finalidad de dar a conocer la realidad existente en 
este centro y su estructura de trabajo, haciendo palpable la posibilidad de trasformar la realidad en las aulas.

\section{Legislación y compesación educativa}

En torno a esta premisa, se enmarca una base legislativa que sustenta este proceso para garantizar el derecho a la igualdad de todos y el derecho a una educación de calidad y en igualdad de oportunidades.

Podemos resumir la legislación en torno a la que gira el concepto de educación compensatoria haciendo mención a las siguientesleyes:

- Ley 9/1999, de 18 de noviembre, de Solidaridad en la Educación. Con esta ley la Junta de Andalucía renovaba y consolida, ya que sigue en vigor, su compromiso con la igualdad de oportunidades, la universalización del derecho a la educación y la integración social de los sectores de población desfavorecidos. El objetivo fundamental de esta ley es art. 1 "Garantizar la solidaridad en la educación, regulando el conjunto de actuaciones que permitan que el sistema educativo contribuya a compensar las desigualdades, asegurando la igualdad de oportunidades al alumnado con necesidades educativas especiales". La finalidad del respaldo de esta ley es poder llevar a cabo programas y actuaciones de carácter general destinados a la compensación educativa y social, con el fin de paliar las desigualdades por diferentes razones que se producen en nuestra sociedad.

- Decreto 167/2003, de 17 de junio, por el que se establece la ordenación de la atención educativa a los alumnos y alumnas con necesidades educativas especiales asociadas a condiciones sociales desfavorecidas.

- $\quad$ Orden de 26 de febrero de 2004, por la que se regula el procedimiento para la elaboración, aprobación, aplicación y evaluación de planes de compensación educativa por los centros docentes sostenidos con fondos públicos.

- Acuerdo de 25 de noviembre de 2003, del Consejo de Gobierno, por el que se aprueba el Plan Integral para la Prevención, Seguimiento y Control del Absentismo Escolar.

Todo este entramado legal se reafirma en la idea de practicar una educación que llegue a todos y de la que todo el alumnado sin distinción sea partícipe. Sin discriminación por su cultura de origen. 


\section{Educación intercultural y escuela inclusiva}

A lo largo de los años esta idea de trabajar la educación por y para todos se ha hecho más fuerte. Asentando sus premisas en pilares como la educación inclusiva. Dando lugar al nacimiento de políticas interculturales y de inclusión, basadas en metodologías de trabajo del profesorado para poder hacer realidad las mismas.

El desarrollo de sistemas más avanzados de enseñanza, trabajando la realidad social es hablar de políticas interculturales y de inclusión pero no solo desde el punto de vista de colectivos desfavorecidos; sino que además es hablar de organización escolar, de currículo, de rendimiento, de didácticas, y diferentes metodologías (Grañeras).

Todo ello al servicio de flexibilizar un sistema donde las estructuras y los currículos sean lo suficientemente dúctiles, con centros con la suficiente autonomía como para organizar el trabajo en función de un objetivo: lograr el éxito escolar y personal de todos y cada uno de sus alumnos.

El hecho de que el alumnado con más barreras para el aprendizaje y la participación no salgan del aula para recibir apoyos adicionales, sino que participen de la vida del aula con los apoyos que necesiten, provoca una mayor integración en el grupo de iguales y una mayor aceptación de las diferencias (Huguet,2009:89).

La educación intercultural es un modo de hacer escuela, un principio rector para educar, para orientar la educación desde el paradigma de la equidad y de la justicia, al servicio de mejorar el papel y la responsabilidad de la escuela en la creación de comunidades democráticas donde cada niña y cada niño crezcan recibiendo lo que necesitan para hacerlo. Los avances son considerables tanto en la ley educativa que rige nuestro sistema como en los desarrollos normativos del conjunto de las administraciones educativas, en los que muchas de ellas están optando por un modelo que entiende que la inclusión en educación pasa por una reestructuración de la organización de la escuela, adoptando medidas dirigidas a una mejor coordinación de los diferentes profesionales, una mayor permeabilidad a la diversidad de problemáticas que se presentan en las aulas, entendiendo el proyecto educativo y el curricular como un proceso en constante cambio que permita adaptarse a la naturaleza cambiante de los alumnos. Una estructura, en resumen, más flexible.

Por otra parte, el propio concepto de atención a la diversidad y las medidas diseñadas para aplicarlo en la escuela han ido evolucionando considerablemente, y la atención a la diversidad se ha ido orientando hacia la modificación del sistema para hacer frente a la diversidad del alumnado, superando la visión anterior, que proponía la integración del alumno considerado diferente en un sistema ya establecido.

La atención educativa específica a colectivos concretos, en este caso al procedente de otros países y culturas, supone un aspecto parcial, aunque necesario e ineludible, que se desprende de ese principio rector, junto con otros muchos mecanismos de atención individual o colectiva que la educación inclusiva ha de garantizar para ofrecer una educación de calidad a cada uno de nuestros alumnos y alumnas. 
La escuela inclusiva es aquella que asume el principio del respeto y reconocimiento a la diferencia del alumnado y que se organiza de una forma flexible, a fin de que pueda atender a toda la diversidad de alumnado existente. Por tanto, no discrimina ni segrega, sino que favorece la integración y combate el fracaso escolar trabajando por el éxito educativo de todos.

La escuela inclusiva es aquella que atiende a cualquier alumno y alumna cuyas diferencias puedan dificultar su proceso de aprendizaje. La escuela inclusiva no enfoca la enseñanza, en la práctica, hacia quienes llegarán al final a los niveles académicos de más prestigio. Es decir, la enseñanza que imparte no es selectiva, no deja por el camino a aquel alumnado que no alcanza los objetivos de cada nivel. (Gallego López y otros).

Para Gallego, la educación intercultural está cada vez más relacionada con la educación inclusiva y considera clave rescatar la aportación a nivel internacional de Mel Ainscow uno de los principales defensores de la inclusión desde la perspectiva de ir más allá de la atención a las personas con necesidades especiales y tener como estrategia englobar a todo el alumnado. Las soluciones no deben aplicarse desde el punto de vista individual según Ainscow sino desde el punto de vista curricular facilitando el apoyo y el aprendizaje de todo el alumnado.

Hay que eliminar el término de necesidades que ha venido asociado durante todos estos años al alumnado con discapacidad - destaca Gallego - y centrarnos en cuales son las barreras de todo tipo existen en los centros educativos y están impidiendo que se hagan prácticas inclusivas, proyectos de centro que incluyan a todos los alumnos y potencien el aprendizaje de todos. (Ainscow en Gallego).

La Junta de Andalucía establece, a través de la legislación y de una serie de programas específicos en los centros educativos, la educación intercultural.

La población escolar andaluza se caracteriza, en la actualidad, por una diversidad social y cultural creciente, lo que ha motivado que, durante los últimos años, en el sistema educativo hayan surgido nuevas necesidades a las que ofrecer respuesta. En este sentido, el centro educativo es un espacio privilegiado para la interacción entre personas de diversas procedencias y culturas, que permite tejer lazos de confianza y respeto por encima de las diferencias (Junta de Andalucía:2011)

La educación inclusiva y la educación intercultural aúnan esfuerzos para superar las diferencias sociales y educativas de nuestro alumnado.

\section{Ejemplo de cultura inclusiva. Colegio Luisa de Marillac (Granada)}

Si todo lo anteriormente expuesto se quedara en simple teoría las esperanzas forjadas durante tanto tiempo en una educación más justa, mas igualitaria, mas equitativa, donde todos los niños y niñas sin distinción alguna reciban la formación que necesitan atendiendo a su ritmo, sus capacidades, su cultura... no sería palpable. Existen 
profesionales entusiasmados con su profesión y dispuestos a dar y formarse continuamente para llevar a sus aulas metodologías y estrategias que ayuden a la igualdad en educación.

Ejemplo de ello es el Colegio Luisa de Marillac, situado en una de las zonas más desfavorecidas de la ciudad de Granada, con la totalidad de alumnado de etnia gitana y rumana, es un referente de trabajo y dedicación por la educación sin exclusión. El paso del tiempo y la realidad del profesorado, del alumnado, de las familias y de las entidades del entorno han hecho posible el cambio y el nuevo reto planteado en el centro: la educación inclusiva. Unido a esto el centro ha puesto en marcha un cambio de metodología, de trabajo con su alumnado de Educación Infantil y Primaria. Llevando a cabo programas de educación intercultural y proyectando todo el proceso a través de un proyecto de trabajo común, el Proyecto Roma.

El centro se adhiere al Proyecto Roma con ilusión y esperanza para conseguir mejorar los procesos cognitivos de aprendizaje potenciando el contexto familiar, escolar y social. El trabajo en las aulas del Colegio Luisa de Marillac pasa ahora por potenciar los procesos lógicos de pensamiento siendo conscientes que el ser humano es un ser que se comunica, siente, piensa y actúa.

El Colegio Luisa de Marillac situado en la zona norte de la ciudad de Granada es un centro de educación compensatoria; nace para paliar y solucionar la situación de desarraigo de varias familias desahuciadas y con graves problemas económicos. Las líneas de actuación en el centro han estado marcadas por el trabajo por y para el alumnado del mismo, el profesorado siempre ha estado preocupado por conseguir la máxima inclusión de los mismos en el colegio y en la sociedad, en su entorno. Teniendo esto muy presente en su trabajo diario, el profesorado hizo saltar la voz de alarma al observar que aún llevando a cabo una labor ardua y costosa con todo el alumnado, estos no conseguían optimizar sus capacidades. En el curso 2006-07 se inicia un cambio metodológico en Educación Infantil apostando por el trabajo por Proyectos de Investigación. De forma paralela comienza a funcionar en el centro un seminario permanente de autoformación para buscar posibles metodologías que les llevara a la mejora de la competencia curricular del alumnado. Este seminario se prolongó durante dos cursos y se nutrió, sobre todo, del trabajo que se estaba llevando a cabo en Infantil y de los excelentes resultados que se estaban obteniendo. Animados por éstos se comienza a trabajar por Proyectos en el primer ciclo de Primaria. Donde los resultados son óptimos y satisfactorios. Desde el Centro de Profesores se les propone acudir a un curso sobre el Proyecto Roma. Desde el primer momento se sintieron identificados con el modelo de escuela inclusiva que dicho proyecto les proponía. Fueron estas razones las que llevaron al centro a incluir en su filosofía de trabajo la inclusión, y mediante la participaron en cursos de formación durante el curso 2009-2010 han ido adquiriendo las líneas y los conocimientos básicos en lo que supone el llamado Proyecto Roma.

La cultura de la escuela está formada por las creencias y convicciones básicas del profesorado y de la comunidad educativa en relación con la enseñanza, el aprendizaje de los alumnos y el funcionamiento del centro. La cultura incluye las normas 
que afectan a la comunidad educativa, los procesos de enseñanza y aprendizaje, los sistemas de comunicación, las relaciones y el tipo de relación que existe entre los docentes (Marchesi y Martín, 2000: 302).

Las ideas que estructuran la escuela inclusiva casan perfectamente con el trabajo anterior del centro y con las principales actuaciones de su claustro de profesores. Es por ello que hacer suyos los principios metodológicos de la escuela inclusiva no es una idea descabellada. El 80\% del profesorado ha aceptado el trabajo hacia la inclusión y se ha puesto manos a la obra en este nuevo reto educativo: El Proyecto Roma.

El cambio en las culturas de los centros es un factor clave para que la escuela pueda responder a las necesidades de la sociedad y para que el alumno disponga de las capacidades necesarias para adaptarse e influir en su entorno Huguet (2009:31)

\subsection{Organización del trabajo dentro del aula}

El cambio conceptual y metodológico de la tarea educativa dentro de las aulas ha supuesto para el profesorado de este centro una reestructuración total de su trabajo. Las clases, según López Melero, M,forjador del Proyecto Roma, se organizan por zonas divididas en:

- Zona de los procesos cognitivos/pensar.

- Zona de los lenguajes/comunicar.

- Zona de la afectividad/amor.

- Zona de la autonomía/acción.

En el centro Luisa de Marillac, y siguiendo los planteamientos del Proyecto Roma, las aulas de Infantil y Primaria se dividen en estas cuatro zonas del cerebro de forma bien diferenciada estructuralmente. Estas zonas, desarrolladas en el orden expuesto, pretenden crear en el niño un proceso lógico de pensamiento que le pueda servir a lo largo de toda su vida, ya que las mismas trabajadas por separado potencian el aprendizaje.

El alumno/a acude al aula sabiendo que va a ayudar al compañero y que el trabajo de todos es importante. Las decisiones que se toman son consensuadas por todos los miembros del equipo y nada se impone por norma, son todas decisiones democráticas. El trabajo se realiza en grupo y todos los miembros participan en el proceso de aprendizaje del otro. El aula se convierte en una situación de estimulación. (Gráfico 1)

\section{Gráfico 1 - Momentos de aula}

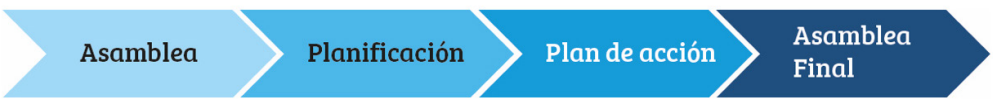

La asamblea es, dentro del Proyecto Roma, el principio y el final de un continuo educativo dentro del aula. Se utiliza como señala López Melero, como estrategia 
metodológica donde se construya cooperativamente el aprendizaje y donde se socializa el mismo. Es en este momento de la clase donde nacen los interrogantes y las hipótesis de trabajo posterior. Lo que se dice en asamblea tiene que ponerse en práctica en todos los momentos de aprendizaje del aula y en la vida cotidiana.

De la asamblea inicial se parte de una representación mental de la situación problemática y se va construyendo con las distintas intervenciones y puntos de vista de cada niño y niña (Cañizares, Pagares y Sánchez, 2005:59)

\subsection{Estrategias de enseñanza y metodología de trabajo}

El profesorado del colegio ha llevado a cabo un proceso de revisión de sus estrategias de enseñanza aprendizaje en el aula al comprobar que sus alumnos no utilizaban todas sus capacidades y la motivación en el aula era escasa. De ahí el Trabajo por proyectos de investigación en el aula ya que:

- Se parte de los conocimientos previos del alumno/a.

- Ellos son protagonistas de su propio proceso de aprendizaje.

- Van construyendo el conocimiento.

- Favorece todo tipo de actividades y agrupamientos.

- Respeta los ritmos de aprendizaje

- Siguiendo el centro de interés del niño/a se escriben y leen todas las palabras relacionadas con el mismo. Por lo que el alumno sigue un proceso lógico y globalizado de la lectura y escritura.

- Se organizan los contenidos en unidades o partes asequibles para los alumnos, presentados con un orden lógico, de modo que puedan ser fácilmente incorporados por el alumno. En las clases y dentro del grupo se organiza la búsqueda de información y esto se lleva a cabo utilizando todos los recursos que se tienen al alcance.

- Se acompaña y apoya a los alumnos en el proceso de conectar los nuevos contenidos con los ya adquiridos. Construcción de aprendizajes significativos y relevantes.

- Utilización del aprendizaje por descubrimiento como el único tipo de aprendizaje que con un alto grado de acierto puede infundirle confianza en sí mismo y muchos de los descubrimientos importantes que realizan los alumnos/as ocurren dentro del contexto de diálogos cooperativos o colaborativos.

- La colaboración con los compañeros en el aprendizaje o en la construcción de los conocimientos, la búsqueda, la indagación, la exploración, la investigación y la solución de problemas, juega un papel importante.

- Se generan materiales y actividades de apoyo, refuerzo y profundización con los que abarcar los diferentes ritmos de aprendizaje. 
- Experimentación con los contenidos, en la medida de lo posible. Investigamos y tratamos de llegar a obtener la información que necesita pasa acceder a los contenidos.

\section{Resultados}

Durante el tiempo transcurrido desde que el proyecto está en marcha el Colegio ha cambiado, reestructurado y revolucionado su forma de actuar y de entender su Proyecto Educativo. Empezando por el equipo de Educación Infantil y pasando por los diferentes ciclos de Educación Primaria, los maestros y maestras se han preocupado por cambiar sus tácticas, procesos y metodologías de enseñanza y están satisfechos con los resultados que actualmente están obteniendo en su alumnado. Los niños y niñas de Infantil llegan al primer ciclo de Primaria con el proceso lector adquirido y continúan con la misma metodología de trabajo con los nuevos profesores del ciclo. El cambio de estrategias, de procesos, de actividades y de proyección del currículum, el trabajo por proyectos, en equipo, respetando la diversidad y aprendiendo de ella está viendo su fruto.

En la actualidad el centro sigue avanzando en su desarrollo y en su implicación en una sociedad más justa, en la cual su alumnado tenga cabida. Con motivo de ello, este pasado curso académico, se han adherido al proyecto de Comunidades de Aprendizaje.(Orden de 8 de junio de 2012, por la que se regula el procedimiento de inscripción y continuidad de centros reconocidos como «Comunidad de Aprendizaje» y se crea la Red Andaluza «Comunidades de Aprendizaje»)

Todas las investigaciones educativas avalan que la participación es un elemento cualitativamente muy relevante en la mejora de los rendimientos y consecuentemente en el éxito escolar. La corresponsabilidad en los aprendizajes implica y desarrolla sentimientos de pertenencia, de vinculación, de colaboración y de compromiso con la mejora. Por ello, fomentar la participación de los diferentes sectores de la comunidad educativa, alumnado, familias y sociedad, es algo más que una moda u oportunidad y se muestra en la actualidad como una condición esencial para la eficacia y el éxito educativo. (Orden de 8 de junio 2012).

El camino comenzado en el Colegio Luisa de Marillac es duro, pero esperanzador, y así lo demuestran los resultados en las aulas. Su afán por aprender unos de otros, su amor por el trabajo bien hecho y por investigar lo que les mueve para su vida diaria es razón más que suficiente para seguir luchando por la inclusión. 


\section{Bibliografías}

\subsection{Libros}

AMARO AGUDO, A. (Coord.) (2010). Fundamentos y principios de la Educación Especial. Aspectos didácticos y organizativos. Madrid: Universitas.

AINSCOW, M. (2001). Desarrollo de escuelas inclusivas. Madrid: Narcea.

BUENDIA EISMAN, L.; COLAS BRAVO, P. y HERNANDEZ PINA, F. (1998). Métodos de investigación en psicopedagogía. Madrid:Mc Graw Hill.

BUENDIA EISMAN, L y COLAS BRAVO, P. (1998). Investigación educativa Ed. Alfar, Sevilla.

JUNTA DE ANDALUCIA. (2011). Guía básica de educación intercultural. Consejería de educación.

LOPEZ MELERO, M. (2004). Construyendo una escuela sin exclusiones. Una forma de trabajar en el aula con proyectos de investigación. Málaga: Aljibe.

MARCHESI, A y MARTÍN, E. (2000). Calidad de la enseñanza en tiempos de cambio.Madrid:Alianza editorial.

7.2 Capítulos o artículos en libros o revistas en papel

ÁVILA CAÑIZARES, R; PARAGES LOPEZ, M y SANCHEZ PALMA M,T. (2005). "Así trabajamos el proyecto Roma en la escuela". En:Cuadernos de Pedagogía, $\mathrm{n}^{\circ}$ 346 MAYO:p. 56-62.

HUGUET, T.(2009). "El trabajo colaborativo entre el profesorado como estrategia para la inclusión."En: La educación inclusiva. De la exclusión a la plena participación de todo el alumnado.Gine, C (coord.), Duran, D.,Font, J y Miguel, E. Cuaderno de Educación, $n^{\circ} 56$.

7.3 Artículos en publicaciones web

AMARO AGUDO, A. (2013) Atención a la diversidad y educación compensatoria. NEAE en el colegio Luisa de Marillac (Granada).[29-09-2013] en: http:// www.amaroagudo.com/atencion-a-la-diversidad-y-educacion-compensatoria-neae-en-el-colegio-luisa-de-marillac-granada

AINSCOW, M. (2004). Salamanca 10 años después: ¿qué impacto ha tenido en el ámbito internacional?. [01-09-2013] en: http://www.educacionespecial.sep.gob. $\mathrm{mx} / \mathrm{pdf} /$ doctos/3Internacionales/5DiezSalamanca.pdf

GRAÑERAS PASTRANA, M. yDÍAZ-CANEJASELA, P.Hacia unas políticas educativas inclusivas: el binomio inmigración-educación intercultural. Red de escuelas interculturales.[22-10-2013] en: www.escuelasinterculturales.eu/spip. php?article79

GALLEGO LÓPEZ, B;MORENO HERRERO, I;CIFUENTES, L.;TUTS, M. yBESALÚ, X.Escuela inclusiva y educación intercultural. Red de escuelas interculturales.[22-10-2013]en: www.escuelasinterculturales.eu/spip.php?article103

LLUCH, X. Educación intercultural y pueblo gitano. Red de escuelas interculturales.[22-10-2013] en:www.escuelasinterculturales.eu/spip.php?article146 


\subsection{Legislación}

Ley 9/1999, de 18 de noviembre, de Solidaridad en la Educación. (BOJA 2/12/1999). Decreto 167/2003, de 17 de junio, por el que se establece la ordenación de la atención educativa a los alumnos y alumnas con necesidades educativas especiales asociadas a condiciones sociales desfavorecidas. (BOJA 23/06/2003).

Orden de 26 de febrero de 2004, por la que se regula el procedimiento para la elaboración, aprobación, aplicación y evaluación de planes de compensación educativa por los centros docentes sostenidos con fondos públicos. (BOJA 16/03/2004).

Orden de 8 de junio de 2012, por la que se regula el procedimiento de inscripción y continuidad de centros reconocidos como «Comunidad de Aprendizaje» y se crea la Red Andaluza «Comunidades de Aprendizaje».

Acuerdo de 25 de noviembre de 2003, del Consejo de Gobierno, por el que se aprueba el Plan Integral para la Prevención, Seguimiento y Control del Absentismo Escolar. (BOJA 05/12/2013).

Ley Orgánica 2/2006, de 3 mayo, de Educación (BOE 4/5/2006).

\section{La autora}

Ana Amaro Agudo. Dentro de mi currículum debo resaltar los siguientes aspectos. Licenciada en Pedagogía en 1998 y Doctora en Pedagogía por la Universidad de Granada en 2002. Tesis doctoral "El movimiento de ciudades educadoras en España: una investigación evaluativa". Docente como PT en un centro de compensatoria 2004-2009, realizando labores de apoyo a la integración. Profesora Titular del centro de Magisterio la Inmaculada adscrito a la Universidad de Granada desde 2008. Coordinadora del área de Didáctica y Organización escolar durante el curso 2011/2012. Profesora del Máster sobre Educación Inclusiva en la Universidad Católica de Valencia San Vicente Mártir. Campo de investigación sobre educación inclusiva en diferentes proyectos. Profesora investigadora a tiempo parcial en grupo de investigación AREA. Departamento de Didáctica y Organización Escolar. Facultad de Ciencias de la Educación de la Universidad de Granada. Miembro del equipo de investigación del Proyecto SECS_EVALNEC (SISTEMA EDUCATIVO DE COHESION SOCIAL: DISEÑO DE UN MODELO DE EVALUACION DE NECESIDADES). Ministerio de Economía y Competitividad. Proyecto I+D. (2013-2015). 